\title{
Serum Ferritin Level in Male with Established Coronary Artery Disease
}

\author{
Chanchal L*, Shaini L**, Sachin Deba Th***, Sangeeta N****, Arpita Das*, \\ Victoria L*, C. Lalrindiki*, M.A Singh*****, Ahilya P****** \\ $P G$ of Dept of Biochemistry, RIMS, India* \\ Associate Professor of Dept of Biochemistry, RIMS, India** \\ Professor of Dept of Cardiology, RIMS, India*** \\ Assistant Professor of Dept of Biochemistry, RIMS, India***** \\ Professor of Department of Biochemistry, RIMS, India****** \\ EX-CMO, Casualty Dept. RIMS, India******
}

\begin{abstract}
:
Background: Elevated body iron stores have been implicated as a risk factor of coronary artery disease (CAD). Aim: To study the association of serum ferritin with CAD [acute myocardial infarction (AMI) and chronic stable angina (CSA)] and to assess the relationship of serum ferritin with established conventional risk factors. Methods: Hospital based cross sectional study, 106 CAD patients $-A M I=66$ and CSA $=40$ and 60 aged matched control were included in the study. Serum ferritin was estimated by ELISA method, C-reactive protein ( CRP) by latex agglutination and other risk factors by enzymatic method. Results: Mean serum ferritin were significantly higher in AMI group than CSA and control group ( $p<0.01)$. Serum ferritin was significantly correlated to total cholesterol (TC) $\left(r=362^{* *}, p<0.01\right)$, triglycerides (TG) $(r=250 *, p<0.05)$, low density lipoprotein $(L D L) \quad(r=379 * *, p<0.01)$, high density lipoprotein $(H D L)(r=-291 * *, p<0.01)$, very low density lipoprotein $(V L D L)\left(r=250^{*}, p<0.05\right)$, body mass index (BMI) $\left(r=400^{* *}, p<0.01\right), C R P(r=419 * *, p<0.01)$. The cut off point of $\geq 199 \mathrm{ng} / \mathrm{ml}$ of serum ferritin to predict the possibility AMI, is recorded with $66.7 \%$ sensitivity and $90 \%$ specifity. Conclusion: High serum ferritin is associated with AMI in Manipuri male population. And the measurement of serum ferritin may be used as a complementary tool for confirming the diagnosis of AMI.

Keywords: Coronary artery disease, iron stores, men, serum ferritin.
\end{abstract}

\section{Introduction}

Coronary artery disease (CAD) is the leading cause of death and disability worldwide. Most cardiovascular events are secondary to atherosclerosis, a disease of the arteries involving a local thickening of the vessel wall. Atherosclerosis represents a state of heightened oxidative stress characterized by lipid and protein oxidation in the vascular wall.[1] Established cardiovascular risk factors, such as dyslipidemia, hypertension, diabetes, and smoking, can initiate endothelial dysfunction by altering the cell redox state in the vessel wall and thus induce atherosclerosis.[2] Iron catalyses the formation of reactive oxygen species through Haber-Weiss reactions. [3] Because serum ferritin concentration is directly proportional to intracellular ferritin concentration, it is currently the best measure of body iron stores.[4]

A possible association between body iron status and risk of coronary heart disease was bolstered from a three year Finnish study.[5]Few studies have been carried out to investigate the relationship of ferritin and risk of CAD in Asian region. Therefore the present study was undertaken with the main objective to estimate serum ferritin level in male patients with coronary artery disease (AMI and CSA) and in normal subjects and also to assess the correlation of serum ferritin with lipid parameters.

\section{Methods}

In this cross-sectional Hospital based study 106 consecutive male patients $\mathrm{AMI}=66$ and $\mathrm{CSA}=40$, age above 18 years with CAD attending RIMS hospital were enrolled in the study. Age matched apparently healthy normal subjects free from any systemic disease were included in the control group. Enrolment started on September 2011 and ended on August 2013. Subjects were excluded if they presented with hemolytic diseases and anaemia, congenital heart diseases, hereditary, hemochromatosis, renal and hepatic impairment, acute or chronic infection, chronic inflammatory conditions, iron and antioxidants supplements.

A detailed history including the patients name, age, duration of disease, age of onset of disease, presence of hypertension, associated symptoms like chest pain, palpitation etc. was taken. Personal history of smoking, consumption of alcohol, presence or absence of obesity and family history of diabetes, hypertension or 
coronary heart disease and medical treatment were also recorded. Baseline investigation data like ECG and Chest x-ray available with the patient were also taken for reference.

\subsection{Ethics statement}

The study protocol was approved by ethics review committee of Regional institute of medical sciences, and a prior written informed consent was obtained from all the study participants.

\subsection{Blood sampling and measurement of biomarkers}

Fasting blood samples were collected within 24 hours of the onset of chest pain. Serum ferritin was assayed using ELISA method kit manufactured by Monobind, U.S. C-reactive protein (CRP) assay was done by latex slide agglutination test method using the reagent kit procedure from Tulip. Total cholesterol (TC), Triglyceride (TG) \& high density lipoprotein (HDL) was done by CHOD PAP method using kits obtained from Human, Germany. Low density lipoprotein (LDL) \& very low density lipoprotein (VLDL) cholesterol was calculated by Friedwald formulae. Estimation of Fasting Blood Sugar was done by Glucose Oxidase (GOD/POD) method using Glucose liquicolor Kit manufactured by HUMAN, Germany.

\subsection{Statistical analysis}

Statistical analysis of the data was performed on SPSS for Windows, Version 16. Appropriate statistical formulae like unpaired $\mathrm{t}$ - test, $\mathrm{F}$ - test (ANOVA) and paired wise comparison was made through Post Hoc Test for continuous variables. Pearson correlation coefficient " $\mathrm{r}$ " was estimated as parametric test to establish relationship between risk markers and serum ferritin. Cut-off levels for serum ferritin were also calculated for CAD. Sensitivity and specificity of ferritin were also calculated independently through receiver operating characteristics (ROC) curve. Apart from it logistic regression analysis was used. The P-values less than 0.05 and 0.01 was used as cut-off for significant and highly significant value respectively.

\section{Results}

Table 1 shows the comparison of parameters considered in the present study. There was no significant difference in age and Haemoglobin ( $\mathrm{Hb}$ ) level among the groups. BMI and mean fasting blood sugar shows increasing trend from normal group to CSA and to AMI group ( $\mathrm{P}<0.01)$. The serum total cholesterol (TC), triglyceride (TG), low density lipoprotein (LDL), high density lipoprotein (HDL) and very low density lipoprotein (VLDL) shows a significant difference among the study group $(\mathrm{p}<0.001)$. Nevertheless, for CRP, the difference was made between AMI and CSA and was found to be significant $(\mathrm{P}<0.001)$ on independent sample t-test.

Table 1: Group-wise distribution of mean \pm SD of clinical and laboratory parameters

\begin{tabular}{|l|l|l|l|l|l|}
\hline \multirow{2}{*}{ Age } & AMI $(\mathrm{n}=66)$ & CSA $(\mathrm{n}=40)$ & Control(n=60) & \multirow{2}{*}{ F- value/t-test } & \multirow{2}{*}{ P-value } \\
\cline { 2 - 4 } & Mean \pm SD & Mean \pm SD & Mean \pm SD & & 0.102 \\
\hline Hb & $59.82 \pm 10.22$ & $63.23 \pm 9.54$ & $58.93 \pm 5.93$ & 2.270 & 0.181 \\
\hline SBP & $12.17 \pm 1.24$ & $11.91 \pm 1.0$ & $11.81 \pm 1.05$ & 1.727 & 0.331 \\
\hline DBP & $128.11 \pm 23.32$ & $127.50 \pm 10.41$ & $124.03 \pm 6.60$ & 1.112 & 0.021 \\
\hline BMI & $78.74 \pm 12.70$ & $84.18 \pm 8.54$ & $80.00 \pm 6.35$ & 3.952 & $<0.001$ \\
\hline FBS & $24.95 \pm 2.94$ & $22.94 \pm 2.12$ & $22.39 \pm 1.94$ & 22.889 & 0.001 \\
\hline TC & $102.23 \pm 40.18$ & $89.82 \pm 25.78$ & $82.11 \pm 12.10$ & 7.651 & $<0.001$ \\
\hline TG & $202.41 \pm 40.47$ & $184.30 \pm 21.52$ & $162.35 \pm 31.77$ & 22.340 & $<0.001$ \\
\hline LDL-C & $170.94 \pm 55.47$ & $167.50 \pm 44.50$ & $132.93 \pm 36.04$ & 11.957 & $<0.001$ \\
\hline HDL-C & $130.09 \pm 38.99$ & $111.25 \pm 21.96$ & $85.67 \pm 33.54$ & 27.539 & $<0.001$ \\
\hline VLDL & $37.20 \pm 9.58$ & $39.55 \pm 6.74$ & $50.53 \pm 11.39$ & 32.252 & $<0.001$ \\
\hline CRP & $35.80 \pm 13.51$ & $33.25 \pm 9.11$ & $27.56 \pm 7.06$ & 9.904 & $<0.001$ \\
\hline
\end{tabular}

Table 2: Post Hoc Test results for multiple comparisons

\begin{tabular}{|c|c|c|c|c|}
\hline Dependent Variable & (1) Group & (2) Group & Mean Difference (1-2) & P-value \\
\hline Ferritin & $\begin{array}{l}\text { AMI } \\
\text { CSA }\end{array}$ & $\begin{array}{l}\text { CSA } \\
\text { Control } \\
\text { control }\end{array}$ & $\begin{array}{l}75.97439(* *) \\
92.42439(* *) \\
16.45000\end{array}$ & $\begin{array}{l}<.001 \\
<.001 \\
0.474\end{array}$ \\
\hline
\end{tabular}


Table 2: Post Hoc Test was applied on serum ferritin, no difference was found between CSA and control for ferritin as their corresponding P-values were not significant. While the difference for other groups were found to be highly significant $(\mathrm{p}<0.001)$.

Table 3: Group- wise correlation coefficient between marker and risk factors

\begin{tabular}{|l|l|}
\hline Pearson Correlation & CAD \\
\hline & Ferritin \\
\hline TC & $362 * *$ \\
\hline TG & $250^{*}$ \\
\hline LDL & $379 * *$ \\
\hline HDL & $-291 * *$ \\
\hline VLDL & $250^{*}$ \\
\hline Diastolic & 014 \\
\hline Systolic & 153 \\
\hline BMI & $400^{* *}$ \\
\hline FBS & 098 \\
\hline CRP & $419 * *$ \\
\hline
\end{tabular}

*The mean difference is significant at 0.05 level

**The mean difference is significant at 0.01 level

Table 3: The correlation coefficient " $r$ " was calculated between ferritin with each parameter for CAD. In CAD group, ferritin was observed to have a significant correlation with TC, TG, LDL, HDL, VLDL, BMI and CRP. All the correlations except HDL were positive.

Table - 4: Results of logistic regression analysis

\begin{tabular}{|l|l|l|l|l|l|l|}
\hline & B & S.E. & Wald & df & P-value & OR \\
\hline Ferritin & 0.014 & 0.004 & 9.819 & 1 & 0.002 & 1.014 \\
CRP & 0.432 & 0.184 & 5.552 & 1 & 0.018 & 1.541 \\
Constant & -2.186 & 0.662 & 10.893 & 1 & 0.001 & 0.112 \\
\hline
\end{tabular}

\section{OR: Odds ratio}

Table-4: Logistic regression analysis was applied to assess the impact of serum ferritin on CAD after adjusting the linear effects of CRP. From this table OR for serum ferritin was found to be 1.014 indicating when one unit increases in serum ferritin there was $1.4 \%$ more chance of having CAD after adjusting the effects of CRP.

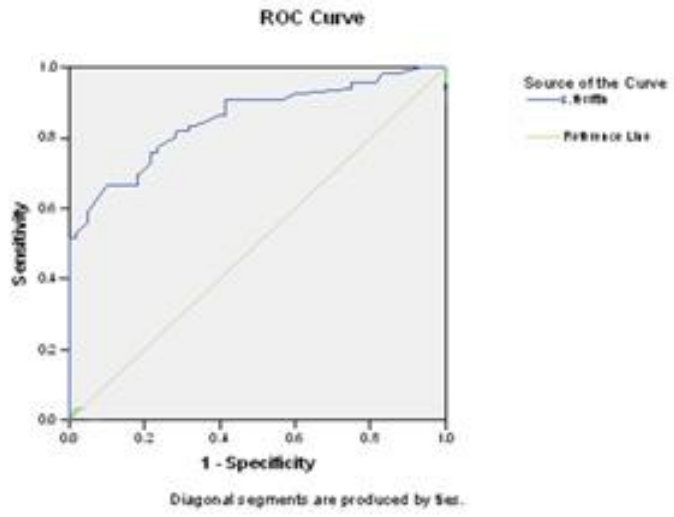

Fig-1: Shows of ROC Curve of serum Ferritin for the prediction of AMI

Area under the ROC curve for ferritin was found to be 0.854 (graphically also shown in fig-1). It indirectly indicates that diagnostic accuracy of serum ferritin to detect $\mathrm{MI}$ is $85.4 \%$. 


\section{Discussion}

This study was restricted to male groups to eliminate the confounding effects of gender. A combined sample of a men and women is hard to interpret given the large gender difference in iron stores. In the present study AMI patients had significantly higher ferritin than CSA and control. Recently evidence of association of elevated serum ferritin and increased risk of AMI came from the studies in Asian ethnic population. $[5,6,7]$

These findings strongly support the "iron hypothesis" which suggests that iron, as a catalytic agent, could promote formation of highly reactive oxygen. The reactive oxygen species (ROS) has been implicated in lipid peroxidation and atherosclerosis subsequently leading to AMI.[8]

However some prospective epidemiologic studies found no association between iron stores and CAD. $[9,10]$ The lack of consistency in the epidemiologic studies is probably explained by the variability in estimates of iron stores, iron intake and by the diversity of study outcomes.[11,12] Again inconsistency between different prospective studies for ferritin may be attributable to differences in genetic factors among different countries.[3] On the other hand Ma J and Stampfer M.J. ${ }^{13}$ opined that lack of association could also reflect bias by factors related to treatment, such as aspirin, or behavioural changes, such as a healthy diet or increase in exercise.

In this study though the serum ferritin level is higher in CSA than the control group, the mean difference is not significant. Lack of association of ferritin with CSA can possibly be due to use of aspirin in CSA patients for long period. Regular daily ingestion of small dose of aspirin, if continued for months or years, may cause a substantial decrease in iron stores.[14]

Serum ferritin being acute phase reactants may not accurately reflect body iron stores in presence of infection or inflammation. To minimize the potential source of confounding, adjustment of CRP, a sensitive marker of inflammation is done in the present study. It is found that for every one unit increase in serum ferritin there is $1.4 \%$ more chance of CAD after adjusting the effects of CRP. Haidari $\mathrm{M}$ et $\mathrm{al}^{3}$ also observed in their study that adjustment of hsCRP did not affect the association between ferritin and CAD.

Again to remove the effect of acute phase of the disease on serum ferritin level all the blood samples were obtained within 24 hours of the onset of chest pain in case of AMI patients. As ferritin levels begin to increase on day 2 following AMI ${ }^{15}$ so it would be reasonable to assume that increased serum ferritin levels in ACS patients reflect body iron stores without any contribution from acute phase response.

In this study it is proposed to set up a serum level of $\geq 199 \mathrm{ng} / \mathrm{ml}$ for serum ferritin $(66.7 \%$ sensitivity and $90 \%$ specificity) as the cut-off to predict AMI. This study shares the limitations of cross-sectional, observational studies. The study can only evaluate the association of ferritin with $\mathrm{CAD}$, not the prospective prediction or causation.

\section{Conclusion}

CAD was positively associated with high serum ferritin in male patients independent of the subclinical inflammation. Measurement of serum ferritin may be used as complementary tool for confirming the diagnosis of AMI. Further prospective and experimental study, are required to corroborate the observed association of ferritin with CAD.

\section{Acknowledgements}

We are indebted to the Department of Biotechnology, Govt of India for funding in this study.

\section{References}

[1]. Stocker R, Keaney JF. Role of oxidative modification in atherosclerosis. Physiol Rev, 84, 2004, $1381-478$

[2]. Morita T. Heme Oxygenase and Atherosclerosis. Arterioscler Thromb Vasc Biol , 25, 2005,1786-95.

[3]. Haidari M, Javadi E, Sanati A, Hajilooi M, Ghanbili J. Association of increased ferritin with premature coronary stenosis in men. Clin Chem, 47(9), 2001, 1666-72.

[4]. Meyers DG, Strickland D, Maloley PA, Seburg JJ, Wilson JE, McMannus BF. Possible association of a reduction in cardiovascular events with blood donation. Heart, 78, 1997, 188-93.

[5]. Delphine W, Silvia CR, Biswas S, Uthappa S, Shetty P. Ferritin a potent threat for acute myocardial infarction. JAPI, 51, 2003, 94750 .

[6]. Iqbal MP, Mehboobali N, Tareen AK, Yakub M, Iqbal SP, Iqbal K et al. Association of Body Iron Status with the Risk of Premature Acute Myocardial Infarction in a Pakistani Population. Plos One, 8(6), 2013,1-5.

[7]. Holay MP, Choudhary AA, Suryawanshi SD. Serum ferritin - a novel risk factor in acute myocardial infarction. Indian Heart Journal, 2012, 173-77.

[8]. De Valk B, Marx JJM. Iron, atherosclerosis, and ischemic Heart disease. Arch Intern Med, 1999, 159, 1542 - 48

[9]. Sempos CT, Looker AC, Gillium RF, Makuf DM. Body iron stores and the risk of coronary heart disease. N Engl J Med, 330,1994, $1119-24$

[10]. Moore M, Folsom AR, Bames RW, Eckfeldt JH. No association between serum ferritin and asymptomatic carotid atherosclerosis. The Atherosclerosis Risk in Communities (ARIC) Study. Am J Epidemiol, 141, 1995,719-23.

[11]. Sempos CT, Looker AC, Gillium RF. Iron and heart disease: the epidemiologic data. Nutr Rev, 54, $1996,73-84$.

[12]. Corti MC, Gaziano M, Hennekens CH. Iron status and risk of cardiovascular disease. Ann Epidemiol , 7, 1997, 62-8.

[13]. Ma J, Stampfer MJ. Body iron stores and coronary heart disease. Clinical Chemistry, 4, 2002, 601-3. 
[14]. Grahan DY, Smith JL. Aspirin and the stomach. Ann Intern Med, 104, 1986, 390-8.

[15]. Moroz C, Bessler H, Katz M, Zahavi I, Salman H, et al. Elevated serum ferritin level in acute myocardial infarction. Biomed Pharmacother 51(3), 1997, 126-30. 